\title{
Frühe Defibrillation beim Herz-Kreislauf- Stillstand: mögliche Strategie für die Schweiz
}

\author{
Cyrill Morger, \\ Martin von Planta, \\ Hans Binz, \\ Martin Luginbühl, \\ Zeno Supersaxo, \\ Stephan Windecker, \\ Hugo Saner
}

\section{Korrespondenz:}

Dr. med. Cyrill Morger

Klinik und Poliklinik für Kardiologie

Departement Herz und Gefässe

Universitätsklinik Inselspital

CH-3010 Bern

cyrill.morger@insel.ch

\section{Einleitung}

Am 8. Juni 2006 ist die Solothurner Nationalrätin Beatrice Heim infolge eines Herz-KreislaufStillstands im Bundeshaus zusammengebrochen. Sie verdankt es den «Ersthelfern» im Nationalratssaal und der frühen Defibrillation, dass sie neurologisch intakt überlebt hat. Dieser Notfall hatte sich im Herzen einer grossen Stadt in einem medizinisch gut ausgerüsteten Gebäude ereignet, es waren Fachleute vor Ort und der Rettungsdienst war schnell zur Stelle - die Voraussetzungen für einen erfreulichen Ausgang waren optimal.

Wenige Tage später kam es in einer kleinen Gemeinde im Kanton Solothurn zu einer ähnlichen Situation: ein 53jähriger Familienvater erlitt zu Hause einen Herz-Kreislauf-Stillstand. Sofort sind der Rettungsdienst und parallel dazu die First Responder (für Herznotfälle ausgebildete Feuerwehrleute) alarmiert worden. Die First Responder erreichten den Patienten innerhalb von vier Minuten und konnten ihn mit dem später eingetroffenen Ambulanzteam erfolgreich reanimieren. Dem Patienten geht es heute gut. Ohne den schnellen Einsatz der First Responder hätte dieses Ereignis weniger erfreulich ablaufen können.

Beide Patienten hatten Glück: Die frühe Defibrillation rettete ihnen das Leben, und sie gehören zu den weniger als $5 \%$ aller Überlebenden eines Herz-Kreislauf-Stillstands. Die Überlebenschancen wären deutlich geringer gewesen, wenn sich die beiden Ereignisse an anderen Orten abgespielt hätten. Trotz dieser eindrücklichen Beispiele ist in der Schweiz die frühe Defibrillation beim Herz-Kreislauf-Stillstand noch immer eine Ausnahme. Dabei ist sie die erfolgversprechendste Methode zur Verhinderung plötzlicher, vorzeitiger Todesfälle bei Kammerflimmern [1]. Nationalrätin Beatrice Heim hat Anfang Oktober 2006 dem Bundesrat eine Motion übergeben, in der sie die Förderung der Nothilfe in der Schweiz und die flächendeckende Versorgung mit Defibrillatoren an ausgewählten Orten fordert. Welche Strategie soll nun gewählt werden, um in der Schweiz den frühen Einsatz halbautomatischer Defibrillatoren mit einem vernünftigen organisatorischen und finanziellen Aufwand zu ermöglichen?

\author{
Défibrillation précoce en cas \\ d'arrêt cardiaque: \\ stratégie possible en Suisse
}

Près de 8000 personnes en Suisse subissent chaque année un arrêt circulatoire en dehors de l'hôpital. Le rythme initial le plus fréquent constaté dans ces accidents est la fibrillation ventriculaire. Malgré les améliorations apportées aux techniques de sauvetage et les nouveautés techniques (p.ex. les défibrillateurs automatiques [AED]), seuls $5 \%-$ comme auparavant - des personnes touchées survivent à l'événement. La raison en est le trop long intervalle précédant l'instauration d'une CPR (réanimation cardiopulmonaire) suffisante et d'une défibrillation, qui devraient être mises en route dans les cinq à huit minutes. La plupart des victimes se trouvent chez elles lors de l'accident. Les services de sauvetage existants (144) ne peuvent raccourcir l'intervalle précité. Un système reposant sur des sauveteurs non professionnels ayant reçu des instructions (first responders) est le mieux à même de compléter les services de sauvetage professionnels. Les cantons de Soleure et du Tessin exceptés, il n'existe jusqu'ici guère de tels systèmes dans le reste du pays. Cette stratégie est financièrement supportable: elle coûte deux francs par année et par habitant dans le canton de Soleure. L'installation d'AED dans des postes fixes, par contre, est certes prometteuse dans les lieux à forte présence humaine, mais elle n'est financièrement pas possible à grande échelle.

\section{Mögliche Strategien}

Die Inzidenz des plötzlichen Herztodes hat in den letzten Jahren abgenommen und beträgt 0,4 bis 1/1000 Personen/Jahr [2-4]. In der Schweiz sind jährlich etwa 8000 Menschen betroffen [1]. Über 80\% dieser Patienten befinden sich in den 
Abbildung 1

Nach einem Kreislaufstillstand mit Kammerflimmern sinkt die Überlebenschance jede Minute bis zur Defibrillation um 10\% [27].

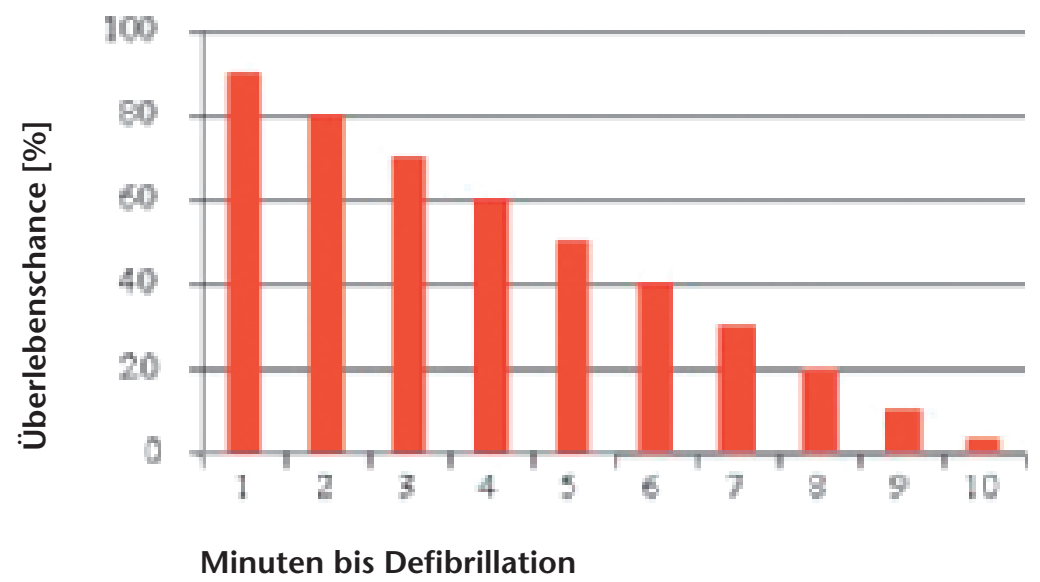

ersten Minuten nach dem Ereignis im Kammerflimmern, das durch frühe Defibrillation mit gutem Resultat behandelt werden kann [5]. Es wird empfohlen, die Defibrillation innerhalb von maximal acht Minuten durchzuführen, am besten innerhalb von fünf Minuten [6]. Die Erfolgschance sinkt beim beobachteten Kreislaufstillstand mit Kammerflimmern ohne kardiopulmonale Reanimation (CPR) jede Minute um $10 \%[7,8]$.

Ein besonders gut organisiertes Rettungssystem hat die Region Seattle (USA), wo in den letzten 25 Jahren die Überlebenschance bei beobachtetem Kreislaufstillstand mit Kammerflimmern im Schnitt um 20\% lag [9]. Grossen Anteil an diesem Erfolg hatte die Sensibilisierung der Bevölkerung für den Rettungsablauf und die Ausbildung weiter Teile der Bevölkerung in der Technik des Basic Life Support (Herzdruckmassage und Beatmung). Die professionellen Rettungsdienste der Schweiz sind nur in einem kleinen Teil der Fälle in der Lage, die Patienten innert fünf bis acht Minuten nach Eintritt des HerzKreislauf-Stillstandes zu erreichen: Die Bemühungen der Rettungsprofis bleiben deshalb oft vergeblich und nur wenige Betroffene überleben (USA: $<5 \%$ Langzeitüberlebende nach HerzKreislauf-Stillstand ausserhalb des Spitals) [5]. In den letzten zehn Jahren sind, speziell für den Einsatz durch medizinische Laien, neue, halbautomatische Defibrillatoren entwickelt worden. Als Alternative zum Vorhalten dieser Geräte an stark frequentierten Orten (PAD - Public Access Defibrillation) besteht die Möglichkeit, diese im Notfall zusammen mit einem ausgebil- deten Team zum Ereignisort zu bringen (mobile Defibrillatoren, sogenanntes First-ResponderSystem). Verschiedene Faktoren wie Alter, erster am Notfallort abgeleiteter Herzrhythmus, Zeit zwischen Zusammenbruch und erster Defibrillation, beobachteter Kollaps und der präklinische Einsatz von Katecholaminen gelten als unabhängige Prädiktoren für den primären Erfolg einer Reanimation sowie für das Langzeitüberleben $[10,11]$.

Für die schnelle Aktivierung der Rettungskette ist zusätzlich der Ereignisort entscheidend. Die erste, im Text beschriebene Situation - der Kreislaufstillstand in der Öffentlichkeit - tritt im Vergleich zum zweiten Beispiel selten auf. 60-70\% der Patienten befinden sich zu Hause, wenn das Ereignis eintritt $[4,12]$.

Auch Patienten mit Herz-Kreislauf-Stillstand zu Hause müssen schnellstmöglich am Ereignisort defibrilliert werden. Dies ist mit dem System der öffentlich installierten Defibrillatoren (PADGeräte) nicht realisierbar. Unkenntnis, fehlende Ausbildung oder Angst vor möglichen Fehlmanipulationen haben zudem oftmals zur Folge, dass öffentliche Defibrillatoren trotz gegebener Indikation gar nicht zum Einsatz gelangen. Die Bedienung ist zwar äusserst einfach, die Hemmschwelle bei nichtausgebildeten Ersthelfern aber dennoch ausgesprochen hoch [13]. Da die Defibrillation innert maximal acht Minuten erfolgen sollte, ist bei öffentlich zugänglichen Defibrillatoren eine sehr hohe Gerätedichte nötig. Da es kaum möglich ist, Hotspots (Orte mit gehäuftem Auftreten von Herz-Kreislauf-Stillständen) mit der erforderlichen Sensitivität zu identifizieren, müssen Gemeinden und Regionen möglichst flächendeckend ausgerüstet werden [14]. Das häufige Auftreten von Herz-Kreislauf-Stillständen zu Hause, die Hemmungen nichtausgebildeter Ersthelfer in der Anwendung sowie die immensen Investitionen für eine flächendeckende Versorgung lassen die Strategie der stationären Defibrillatoren als zu aufwendig und auch für dichtbesiedelte Regionen als ungeeignet erscheinen [15]. Ausgebildete Helfer sind dagegen in der Lage, die Notfallsituationen zuverlässig zu beurteilen und die Geräte richtig einzusetzen [16].

\section{Mobile halbautomatische Defibrillatoren (First Responder)}

First Responder sind für Notfallsituationen ausgebildete und ausgerüstete Ersthelfer, die die Zeit bis zum Eintreffen eines Rettungsdienstes mit Basismassnahmen (Basic Life Support [BLS]) überbrücken. Sie sind in einem Alarmsystem eingebunden und können bei Bedarf parallel zum Rettungsdienst aufgeboten werden. Typischer- 
weise handelt es sich bei First Respondern um Feuerwehrleute, Samariterangehörige oder Polizisten [17]. Ursprünglich ist man von der Idee ausgegangen, dass First-Responder-Teams in denjenigen Regionen eingesetzt werden sollten, die der professionelle Rettungsdienst erst nach langer Anfahrt erreicht, aber in denen die zu geringe Notfallhäufigkeit die Einrichtung eines zusätzlichen Ambulanzstützpunktes nicht rechtfertigt. Inzwischen hat sich gezeigt, dass auch Patienten mit Herz-Kreislauf-Stillstand in einer dichtbesiedelten städtischen Agglomeration durch den Rettungsdienst nicht immer im erforderlichen engen Zeitfenster von 4 bis 8 Minuten erreicht und defibrilliert werden können, was sich zwangsläufig negativ auf die Überlebenschancen auswirkt [18]. Das Einsatzgebiet der FirstResponder-Teams braucht somit nicht auf Randregionen beschränkt zu bleiben.

Dank der gut ausgebauten Notfallversorgung in der Schweiz bestehen zahlreiche, noch nicht ausgeschöpfte Möglichkeiten zur Etablierung der frühen Defibrillation. Die Strategie der mobilen Geräte lässt sich durch Einbeziehen der bereits vorhandenen Milizorganisationen wie

Abbildung 2

Ereignisorte der Notfälle im Frühdefibrillationsprojekt des Kantons Solothurn, n=1200 [28].

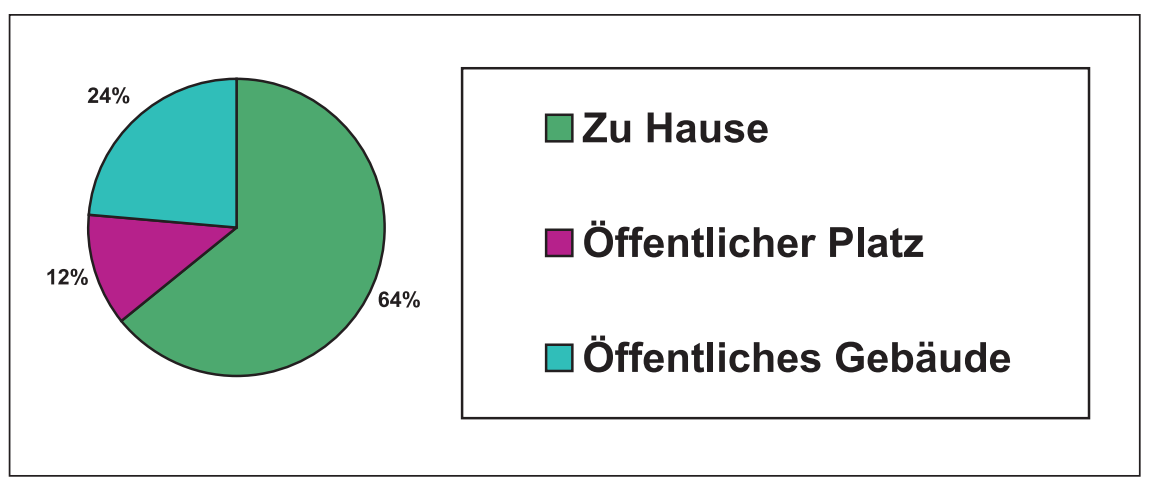

Abbildung 3

Zuerst eingetroffene Helfer bei den Einsätzen des Frühdefibrillationsprojektes im Kanton Solothurn, $n=1200$ [28].

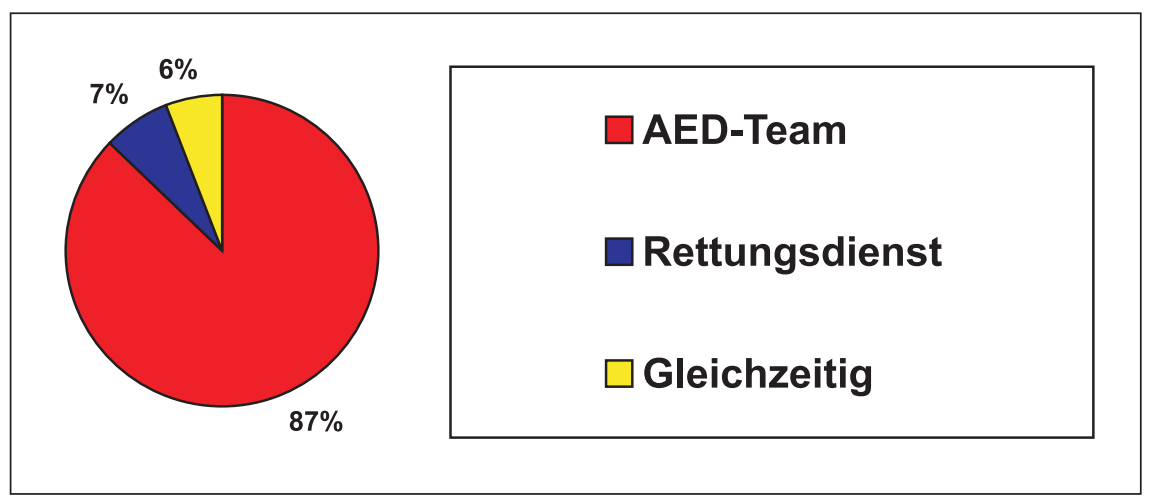

Feuerwehr und Samariter einfach umsetzen. Eine Ausdehnung auf professionelle Dienste wie Notfallärzte oder Polizei ist je nach den regionalen Verhältnissen denkbar. Als wichtigstes Prinzip ist zu beachten, dass die frühe Defibrillation an bereits bestehende Organisationen gekoppelt wird. Damit lassen sich bei Alarmierung, Ausbildung und Ausrüstung Synergien nutzen und Kosten sparen. Durch die dezentrale Organisationsform lassen sich Lücken in der Notfallversorgung schliessen, die durch die Topographie bedingt oder durch die Zusammenlegung bzw. Schliessung von Rettungsstützpunkten entstanden sind. Die mobilen Defibrillatoren müssen während 24 Stunden jeden Tag verfügbar sein. Die professionellen Hilfsdienste wie Polizei, Sanität und Feuerwehr gewährleisten diese Voraussetzung. Feuerwehrleute haben diese Aufgabe bisher meistens besser gelöst als Polizisten [19]. Mit geringem Aufwand kann ihre Logistik für diesen Zweck eingesetzt werden. Kritiker sprechen hier (beispielsweise im Falle der Feuerwehr) von Zweckentfremdung eines bereits überlasteten (Miliz-)Systems. Dem ist entgegenzuhalten, dass bei Notfallorganisationen der Rettung von Menschenleben nicht nur vom Gesetz her, sondern auch aus ethischen Überlegungen oberste Priorität zukommt. Das erweiterte Einsatzspektrum kann durch Delegieren weniger dringender Aufgaben an andere Organisationen aufgefangen werden (z.B. Insektenbekämpfung durch Feuerwehren). Die Angehörigen von Milizorganisationen sind zum Teil in den Basismassnahmen (BLS) ausgebildet und benötigen für ihren Einsatz als medizinische First Responder lediglich eine Zusatzausbildung (z.B. Handhabung des halbautomatischen Defibrillators). Notfallorganisationen kleinerer Gemeinden müssen häufig nur wenige Ernstfalleinsätze leisten. FirstResponder-Teams bieten an diesen Orten eine attraktive Einsatzmöglichkeit für engagierte Helfer. Kurze Reaktionszeiten und ein hohes Mass an Professionalität bei Ausbildung und im Einsatz sind dabei typische Merkmale der vielerorts erfolgreichen Milizsysteme.

\section{Erfahrungen aus dem Kanton Solothurn}

Um die Überlebenschance beim Herz-KreislaufStillstand ausserhalb des Spitals zu verbessern, wird im östlichen Teil des Kantons Solothurn bei Herznotfällen seit sechs Jahren ein First-Responder-Team (Feuerwehrleute und Samariter) durch die Sanitätsnotrufzentrale 144 alarmiert. Der Einsatz des First-Responder-Teams erfolgt basierend auf einer Indikationenliste parallel zum Rettungsdienst. Die Alarmzentrale Solothurn als integrierte Leitstelle ermöglicht die schnelle 
Alarmierung aller involvierten Helfer. Eine kleine, in BLS und halbautomatischer Defibrillation ausgebildete Gruppe von zwei bis vier Personen genügt. Erfreulicherweise sind in einzelnen Gemeinden auch niedergelassene Ärzte in diesen Teams aktiv. Bisher sind rund 1200 Einsätze geleistet worden. Dank guter Ortskenntnis und kleiner Einsatzgebiete sind die Teams durchschnittlich innerhalb von vier Minuten nach der Alarmierung beim Patienten.

Gegen 20 Prozent der Patienten waren reanimationspflichtig. Ein Fünftel von ihnen konnte primär erfolgreich reanimiert werden, $6 \%$ der reanimationspflichtigen Patienten konnten mit guter Lebensqualität nach Hause entlassen werden. Um direkt ein Leben zu retten, muss jedes Herznotfallteam der über 30 Feuerwehren insgesamt dreimal ausrücken. Befürchtungen, dass infolge der frühen Defibrillation vermehrt Patienten mit schweren neurologischen Defiziten überleben, konnten klar widerlegt werden. In den Jahren vor Einführung der First-ResponderTeams hat in der gleichen Region kein Patient einen Herz-Kreislauf-Stillstand ausserhalb des Spitals überlebt. Neben dem First-ResponderKonzept wurde im Kanton Solothurn auch der Bahnhof Olten (Personenfrequenz: 36000/Tag) mit einem AED ausgerüstet, der in den letzten sechs Jahren weniger als zehnmal eingesetzt wurde, sich aber in einem Fall als lebensrettend erwies [4]. Weiter gibt es in der Region Olten zahlreiche AED-Geräte in öffentlichen und privaten Institutionen. Diese sind nicht registriert und stehen der Alarmzentrale bei Notfällen daher nicht zur Verfügung. Besonders in kleinen Gemeinden ist allerdings zu berücksichtigen, dass Einsätze, bei denen sich Helfer und Patient kennen, psychisch belastend sein können. In besonders schwierigen Situationen sollte deshalb mit professioneller Unterstützung eine Einsatznachbesprechung durchgeführt werden [20].

Als schwächstes Glied der Rettungskette erweisen sich immer wieder die ungenügenden Kenntnisse der Bevölkerung in bezug auf Symptome eines Herznotfalles und der richtigen Alarmierung. Bei zahlreichen Patienten, die trotz des schnellen Einsatzes von First Respondern und Rettungsdienst nicht gerettet werden konnten, spielte die verzögerte Alarmierung eine entscheidende Rolle. Aufklärungskampagnen für die Bevölkerung, wie das im Januar 2007 lancierte Projekt HELP der Schweizerischen Herzstiftung, werden in diesem Bereich hoffentlich Verbesserungen ermöglichen (www.helpbyswissheart.ch).

Aufgrund der positiven Erfahrungen mit dem Pilotversuch ist im Kanton Solothurn geplant, das First-Responder-System auf freiwilliger Basis in den Feuerwehren zu etablieren. Es wird dabei den einzelnen Gemeinden überlassen sein, ob sie ihre Feuerwehr mit dieser zusätzlichen Aufgabe betrauen wollen. Die Einführung des FirstResponder-Systems verursacht Investitionen von rund einer halben Million Franken. Die jährlich wiederkehrenden Kosten (Einsätze, Schulungen, Material) belaufen sich auf den gleichen Betrag oder umgerechnet auf zwei Franken pro Einwohner und Jahr. Damit kann den 251000 Menschen des Kantons Solothurn im Notfall sehr schnell geholfen werden. In der Schweiz verfügt neben dem Kanton Solothurn einzig das Tessin über eine weit fortgeschrittene First-Responder-Organisation. In den Kantonen St. Gallen, Thurgau, Wallis und Zürich bestehen vereinzelte FirstResponder-Gruppen.

\section{Erfahrungen mit stationären Defibrillatoren}

Zahlreiche Studien aus Europa sowie den USA haben gezeigt, dass strategisch verteilte Defibrillatoren die Überlebenschancen der Patienten bis auf 74\% (beobachtetes Ereignis, initiales Kammerflimmern, Defibrillation innert weniger Minuten) erhöhen können [21, 22]. Die meisten dieser Arbeiten haben ein engbegrenztes Gebiet untersucht, wo eine Abdeckung von Hotspots mit öffentlich zugängigen Geräten mit dem Ziel möglichst kurzer Interventionszeiten sinnvoll ist [22-24]. Die Herausforderung liegt darin, diese Hotspots zuverlässig zu identifizieren [14, 25]. Dazu kann beispielsweise die Bevölkerungsdichte verwendet werden [4]. Der verlässlichste Hotspot ist die Wohnung des Patienten, wo er sich in über zwei Dritteln der Fälle aufhält, wenn das Ereignis eintritt [12]. Dank sinkender Preise verbreiten sich die halbautomatischen Defibrillatoren mittlerweile auch in Praxen notfalldienstleistender Kollegen sowie in Vereinen (z. B. Samariter), an Arbeitsplätzen (z.B. Bürogebäuden) und in Transportmitteln (z.B. Passagierflugzeugen). Häufig schaffen mehrere Ärzte oder Institutionen solche Geräte gemeinsam an. Insbesondere in den USA werden vermehrt AEDGeräte für Risikopatienten zu Hause abgegeben («home defibrillation» [26]). Dieser Anwendungsbereich ist auf Patienten mit bereits bekannter Risikokonstellation beschränkt. Insgesamt muss aber betont werden, dass mit stationären Defibrillatoren nur ein Drittel der Betroffenen erreicht werden.

\section{Empfehlungen für die Schweiz}

- Trotz beschränkter finanzieller Ressourcen sind wir verpflichtet, die Überlebenschancen von Patienten mit Herz-Kreislauf-Stillstand 
Tabelle 1

Verschiedene Frühdefibrillationssysteme im Vergleich.

\begin{tabular}{lllcl} 
Referenz & Autor & Ort & Anzahl Patienten & \% Spitalentlassung \\
\hline Circulation. 1997;96:2849 & O'Rourke & Inflight/Terminal & $6 / 17$ & $33 / 24$ \\
\hline Circulation. 2000;102:1780 & Schneider & Notfallsystem & 54 & 31 \\
NEJM. 2000;343:1206 & Valenzuela & Spielkasino & 90 & 59 \\
NEJM. 2000;343:1216 & Page & Inflight & 99 & 40 \\
NEJM. 2004;347:1242 & Caffrey & Terminal & 21 & 48 \\
NEJM. 2004;351:637 & PAD & Gemeindebasiert & 28 & 23 \\
Total & & & 310 & 42
\end{tabular}

ausserhalb des Spitals zu verbessern. Nach aktuellem Wissensstand bieten sich dafür flächendeckende First-Responder-Systeme als Ergänzung zum bestehenden Rettungsdienst als realisierbare und erfolgversprechende Variante an. Es handelt sich um eine besonders kostengünstige medizinische Intervention. Die Verantwortlichen für das Rettungswesen in den Kantonen und die Politik sind gefordert, dem erkannten Nachholbedarf in der Schweiz aktiv zu begegnen. Die Strategie, geeignete Helfer und die notwendige Technik sind vorhanden - nutzen wir sie!

- Zusätzlich können definierte öffentliche Hotspots sowie private Bereiche mit automatischen Defibrillatoren ausgerüstet werden, sofern ausgebildete Helfer vorhanden sind. Eine wichtige Aufgabe für die Zukunft ist die Identifikation von Hotspots in der Schweiz durch die präzise Dokumentation der Ereignisorte. Parallel dazu ist eine regionale Erfassung der bereits vorhandenen AED-Geräte wünschenswert, damit diese durch die Sanitätsnotrufzentralen im Notfall berücksichtigt und disponiert werden können.

- Der wiederholten Aufklärung der Bevölkerung über die Symptome eines Herznotfalls, über die korrekte Alarmierung und das Durchführen des Basic Life Support kommt weiterhin eine zentrale Bedeutung zu.

\section{Literatur}

1 Katz E, et al. [What do we actually know about out-of-hospital cardiac arrest?]. Rev Med Suisse. 2005;1(9):628-30, 632-3.

2 Atwood C, et al. Incidence of EMS-treated outof-hospital cardiac arrest in Europe. Resuscitation. 2005;67(1):75-80.

3 Herlitz J, et al. Resuscitation in Europe: a tale of five European regions. Resuscitation. 1999;41(2):121-31.
4 Meier P, Morger C. Der plötzliche Herztod in der Region Olten und Möglichkeiten zur Verbesserung der Überlebensrate: Grundlagen zur AED-Studie. Inauguraldissertation Medizinische Fakultät Universität Bern, 2001.

5 Terranova P, et al. Early outcomes of out-of-hospital cardiac arrest after early defibrillation: a 24 months retrospective analysis. Indian Pacing Electrophysiol J. 2006;6(4):194-201

6 De Maio VJ, et al. Optimal defibrillation response intervals for maximum out-of-hospital cardiac arrest survival rates. Ann Emerg Med. 2003; 42(2):242-50.

7 Klingenheben T. [Resuscitation in ventricular fibrillation: what is essential?]. Herzschrittmacherther Elektrophysiol. 2005;16(2):78-83.

8 Valenzuela TD, et al. Estimating effectiveness of cardiac arrest interventions: a logistic regression survival model. Circulation. 1997;96(10):3308-13.

9 Rea TD, et al. Temporal trends in sudden cardiac arrest: a 25-year emergency medical services perspective. Circulation. 2003;107(22):2780-5.

10 Lindholm DJ, Campbell JP. Predicting survival from out-of-hospital cardiac arrest. Prehospital Disaster Med. 1998;13(2-4):51-4.

11 Bunch TJ, et al. Admission predictors of in-hospital mortality and subsequent long-term outcome in survivors of ventricular fibrillation out-of-hospital cardiac arrest: a population-based study. Cardiology. 2004;102(1):41-7.

12 Muller D, Agrawal R, Arntz HR. How sudden is sudden cardiac death? Circulation. 2006; 114(11):1146-50.

13 Maisch S, Friederich P, Goetz AE. [Public access defibrillation: Limited use by trained first responders and laymen.]. Anaesthesist. 2006.

14 Frank RL, et al. The locations of nonresidential out-of-hospital cardiac arrests in the City of Pittsburgh over a three-year period: implications for automated external defibrillator placement. Prehosp Emerg Care. 2001;5(3):247-51.

15 Clare C. Do public access defibrillation (PAD) programmes lead to an increase of patients surviving to discharge from hospital following out of hospital cardiac arrest? - A literature review. Int J Nurs Stud. 2006;43(8):1057-62. 
16 Morger C, Gaillet R, von Planta M, Saner H. Use of semiautomatic defibrillators by minimally trained lay persons in cardiac emergencies: is it safe? JACC. 2006;47(4 Suppl 1):A1-A478.

17 Trappe HJ. [First responder defibrillation in the USA, Europe and Germany - prerequisites, experiences, perspectives]. Herzschrittmacherther Elektrophysiol. 2005;16(2):94-102.

18 Stotz M, et al. EMS defibrillation-first policy may not improve outcome in out-of-hospital cardiac arrest. Resuscitation. 2003;58(3):277-82.

19 Groh WJ, et al. Limited response to cardiac arrest by police equipped with automated external defibrillators: lack of survival benefit in suburban and rural Indiana - the police as responder automated defibrillation evaluation (PARADE). Acad Emerg Med. 2001;8(4):324-30.

20 Morger C, Gaillet R, von Planta M, Saner H. Combined community based and on site responder AED-system in Switzerland: 10 fold increased long term survival rate of OHCA. JACC. 2005;45(3 Suppl 1):A1-A555.

21 Davies CS, et al. A national programme for on-site defibrillation by lay people in selected high risk areas: initial results. Heart. 2005;91(10):1299-302.
22 Valenzuela TD, et al. Outcomes of rapid defibrillation by security officers after cardiac arrest in casinos. N Engl J Med. 2000;343(17):1206-9.

23 Bartimus HA, Rea TD, Eisenberg MS. Prevalence of automated external defibrillators at cardiac arrest high-risk sites. Prehosp Emerg Care. 2004;8(3): 280-3.

24 Caffrey SL, et al. Public use of automated external defibrillators. N Engl J Med. 2002;347(16):1242-7.

25 Pell JP, et al. Potential impact of public access defibrillators on survival after out of hospital cardiopulmonary arrest: retrospective cohort study. BMJ. 2002;325(7363):515

26 Sanna T, et al. Home defibrillation: a feasibility study in myocardial infarction survivors at intermediate risk of sudden death. Am Heart J. 2006; 152(4):685 e1-7.

27 Cummins RO. From concept to standard-of-care? Review of the clinical experience with automated external defibrillators. Ann Emerg Med. 1989; 18(12):1269-75.

28 Morger C, Gaillet R, von Planta M, Saner H. Early lay defibrillation in Switzerland: the Olten AEDstudy. Article in Press, 2007. 